6. Koch PJ, Franke WW. Desmosomal cadherins: another growing multigene family of adhesion molecules. Curr Opin Cell Biol. 1994;6(5):682-687.

7. Harmon RM, et al. Desmoglein-1/Erbin interaction suppresses ERK activation to support epidermal differentiation. J Clin Invest. 2013 123(4):1556-1570.

8. Stanley JR, Amagai M. Pemphigus, bullous impetigo, and staphylococcal scalded skin syndrome. NEngl J Med. 2006;355(17):1800-1810.

9. Ishii K, Lin C, Siegel DL, Stanley JR. Isolation of pathogenic monoclonal anti-desmoglein 1 human antibodies by phage display of pemphigus foliaceus autoantibodies. J Invest Dermatol. 2008; 128(4):939-948.

10. Wu H, et al. Protection of neonates against pemphigus foliaceus by desmoglein 3. N Engl J Med. 2000 343(1):31-35.

11. Elias PM, et al. Desmoglein isoform distribution affects stratum corneum structure and function. J Cell Biol. 2001;153(2):243-249.
12. Brennan D, et al. Suprabasal Dsg2 expression in transgenic mouse skin confers a hyperproliferative and apoptosis-resistant phenotype to keratinocytes. J Cell Sci. 2007;120(pt 5):758-771.

13. Getsios S, et al. Desmoglein 1-dependent suppression of EGFR signaling promotes epidermal differentiation and morphogenesis. J Cell Biol. 2009; 185(7):1243-1258.

14. Rickman $\mathrm{L}$, et al. N-terminal deletion in a desmosomal cadherin causes the autosomal dominant skin disease striate palmoplantar keratoderma. Hum Mol Genet. 1999;8(6):971-976.

15. Wan H, et al. Striate palmoplantar keratoderma arising from desmoplakin and desmoglein 1 mutations is associated with contrasting perturbations of desmosomes and the keratin filament network. BrJ Dermatol. 2004;150(5):878-891.

16. Lebeau $S$, et al. Comparative analysis of the expression of ERBIN and Erb-B2 in normal human skin and cutaneous carcinomas. Br J Dermatol. 2005; 152(6):1248-1255
17. Matsunaga-Udagawa R, et al. The scaffold protein Shoc2/SUR-8 accelerates the interaction of Ras and Raf. J Biol Chem. 2010;285(10):7818-7826.

18. Siegel DH, Mann JA, Krol AL, Rauen KA. Dermatological phenotype in Costello syndrome: consequences of Ras dysregulation in development. $\mathrm{BrJ}$ Dermatol. 2012;166(3):601-607.

19. Nava C, et al. Cardio-facio-cutaneous and Noonan syndromes due to mutations in the RAS/MAPK signalling pathway: genotype-phenotype relationships and overlap with Costello syndrome. J Med Genet. 2007;44(12):763-771.

20. McGrath JA. Inherited disorders of desmosomes. Australas J Dermatol. 2005;46(4):221-229.

21. Dusek RL, Attardi LD. Desmosomes: new perpetrators in tumour suppression. Nat Rev Cancer. 2011; 11(5):317-323.

22. Tada H, Hatoko M, Tanaka A, Kuwahara M, Muramatsu T. Expression of desmoglein I and plakoglobin in skin carcinomas. J Cutan Pathol. 2000; 27(1):24-29.

\title{
WNT signaling in stem cell differentiation and tumor formation
}

\author{
Hong Ouyang, ${ }^{1,2}$ Yehong Zhuo, ${ }^{3}$ and Kang Zhang ${ }^{1,2,3,4}$
}

\begin{abstract}
${ }^{1}$ Molecular Medicine Research Center and Department of Ophthalmology, State Key Laboratory of Biotherapy, West China Hospital, Sichuan University, Chengdu, People's Republic of China. ${ }^{2}$ Department of Ophthalmology and Shiley Eye Center and Institute for Genomic Medicine, UCSD, San Diego, California, USA. ${ }^{3}$ State Key Laboratory of Ophthalmology, Zhongshan Ophthalmic Center, Sun Yat-sen University, Guangzhou, People's Republic of China. ${ }^{4}$ Veterans Administration Healthcare System, San Diego, California, USA.
\end{abstract}

\begin{abstract}
Embryonic stem cells (ESCs) hold great therapeutic promise for the regeneration of functional cell types and clinical applications. However, tumorigenic potential of stem cells in a transplanted host remains a major obstacle. In this issue of the JCI, Cui and colleagues identified TCF7-mediated canonical WNT signaling as a critical determinant of both the tumorigenicity and therapeutic function of ESC-derived retinal progenitor cells (ESC-RPCs). Their findings suggested that addressing key extracellular signaling and related intrinsic factors will be essential for the successful use of ESC-derived progenitor transplantation.
\end{abstract}

Photoreceptor degeneration underlies major causes of blindness, including macular degeneration and retinitis pigmentosa, affecting tens of millions of people worldwide. In theory, vision of affected patients may improve if the diseased cells (rods and cones) are replaced with new, healthy cells that form appropriate connections with the host retina. Embryonic stem cells (ESCs) possess unlimited self-renewal capabilities and the ability to differentiate into any adult cell type (1). These unique features make ESC-based therapy appealing for the treatment of various degenerative disorders but may also cause unwanted serious

Conflict of interest: The authors have declared that no conflict of interest exists.

Citation for this article: J Clin Invest. 2013; 123(4):1422-1424. doi:10.1172/JCI69324. side effects. It has been previously demonstrated that ESCs or ESC-derived progenitors spontaneously form tumors upon transplantation in vivo, even when the cells are predifferentiated or presorted (2). Despite this issue, successful cell replacement of human ESC-derived (hESC-derived) retinal cells for vision restoration in animal models of photoreceptor degeneration has been reported recently. Photoreceptor precursors derived from hESCs have been shown to migrate into Crx-deficient mouse retina following intraocular injection, express appropriate markers for both rod and cone photoreceptors, and subsequently restore some light responses (3). In another study, subretinal transplantation of hESC-derived retinal pigment epithelium in patients with Stargardt's macular dystrophy and dry age-related macular degeneration improved visual acuity, with no signs of hyperproliferation or tumorigenicity after 4 months (4). These examples illustrate purity, stability, and proper localization of transplanted cells in vivo and prompted the development of numerous differentiation protocols. Still, the risk of tumor formation remains a barrier, because there are no parameters to quantify safety factors and because the appropriate stage of differentiation at which ESC-derived progenitors should be used has not been well evaluated. In this issue, Cui and colleagues attempted to address the above concerns by identifying major extracellular signaling and intrinsic factors controlling tumorigenicity and therapeutic potential of ESC-derived retinal progenitor cells (ESC-RPCs) (5).

\section{ESC tumorigenic potential}

The best proof of pluripotency of ESCs is their ability to form teratomas in which the stem cells differentiate to various tissue types of the embryo in a disordered fashion following transplantation into immunosuppressed mice. Teratomas usually contain all three germ layers and have typical tumor characteristics (6). In other words, ESCs are naturally tum- 


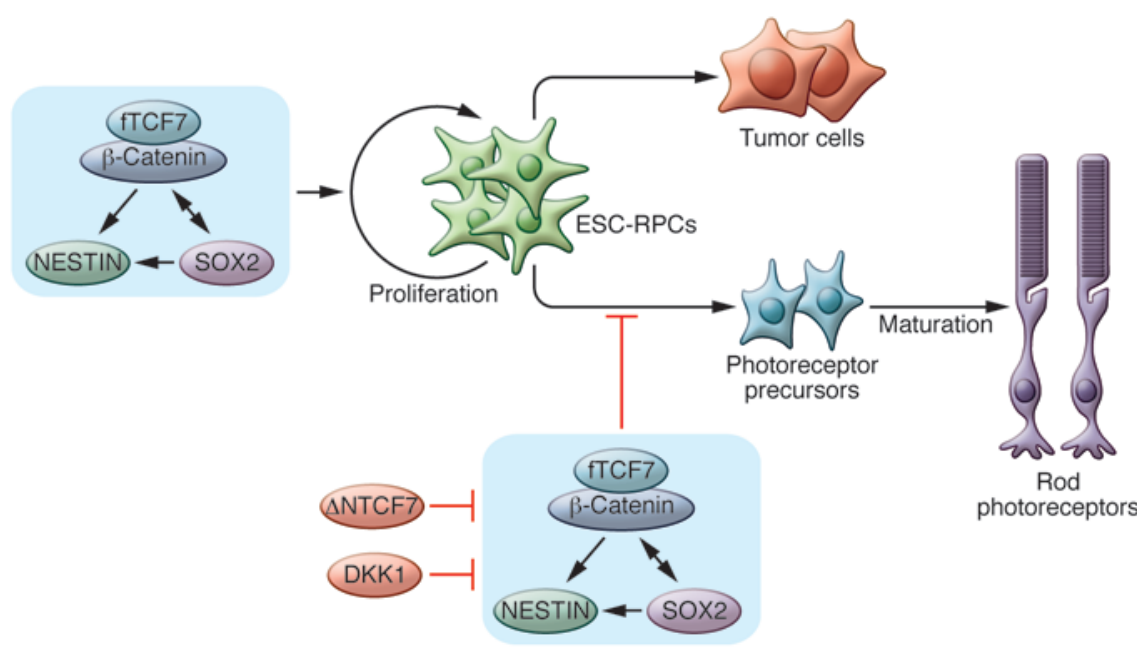

origenic: like cancer cells, they are capable of unlimited proliferation potential and clonal propagation without anchorage dependence. Indeed, tumor formation is commonly considered to be a consequence of hyperproliferation of residual ESCs or precursor cells. Accordingly, purification of tissue-committed progenitor cells from undifferentiated ESCs or removal of undifferentiated ESCs before transplantation becomes a necessary prerequisite in order to reduce the risk of tumor growth. Several strategies have been implemented to address this critical issue, including the selection of specified cells based on their surface markers (e.g., by FACS or magnetic-activated cell sorting), isolation of lineages using genetic manipulation (e.g., lineage-promoter-driven antibiotic resistance), or the knockdown of or pharmacological interference with intracellular signaling pathways that increase tumorigenicity.

\section{Role of WNT signaling in tumorigenesis and differentiation}

Cui et al. demonstrated that ESC-RPCs had a high propensity for neural tumor formation following ocular transplantation (rate 60.58\%); however, transplantation of primary retinal progenitor cells (P-RPCs) from neonatal mice resulted in efficient integration to host retina with no evidence of tumor development. Transcriptomic profiling revealed increased activity of the WNT signaling pathway in ESC-RPCs compared with that in P-RPCs (5). WNT signaling is one of the key signaling pathways in regulating cell proliferation, motility, and differentiation as well as tumorigenesis, and upregulation of $\beta$-catenin, an intracellular mediator of the pathway, is frequently associated with the differentiation potential of ESCs in teratomas (7). To determine whether WNT activation was related to the increased tumorigenicity of ESC-RPCs, Cui and colleagues treated the ESC-RPCs with DKK1, an extracellular inhibitor of WNT signaling, prior to transplantation. Expression profiling revealed that neural progenitor markers and cell proliferation markers were repressed, whereas large number of committed retinal markers were upregulated by WNT inhibition. The pretreatment with DKK1 dramatically suppressed tumor formation and improved integration of ESC-RPCs into the transplanted host retina (5).

Cui et al. further demonstrated that upregulation of the direct transcriptional targets of canonical WNT signaling, NESTIN and SOX2 was critical to the tumorigenicity of ESC-RPCs following transplantation (Figure 1). The authors also confirmed a previous study demonstrating the importance of the WNT pathway in eye development, in which a high percentage of embryonic eye field-specific cells were generated from hESCs by upregulation of DKK1, and the addition of WNT3A and BMP4 to cultures abolished the expression of PAX6 and RAX (8). Notably, a report that WNT/ $\beta$-catenin signaling promotes dedifferentiation and proliferation of Muller glia-derived retinal progenitors and neural regeneration after damage (9) also suggested that sustained activation of the WNT pathway may maintain the proliferation ability of ESC-RPCs and contribute to the potential of tumor formation upon transplantation.

\section{Figure 1}

Canonical WNT signaling determines the proliferation and differentiation state of ESC-derived progenitors via full-length TCF7 (fTCF7). SOX2 and NESTIN act as direct targets of fTCF7 in vitro. Blocking the pathway by deleting TCF7 or treating cells with the WNT inhibitor DKK1 could reduce tumorigenicity and improve therapeutic efficiency. $\triangle N T C F 7$, naturally truncated form of TCF7.

\section{Differentiation of ESCs into retinal progenitor cells}

Despite the concern of tumorigenic potential, stem cell-based therapy still holds the most promise to restore lost vision in patients with retinal diseases, due to our extensive knowledge of retinal development (10). To date, a number of protocols have been developed for differentiation of mature retinal cells, including photoreceptors, by a stepwise treatment with defined factors $(3,8)$. Notably, the rates of tumor formation and successful host integration vary among different reports, even using a same selective marker. Cui et al. selected SOX1.EGFP-positive neural progenitor cells first and then obtained ESC-RPCs and subsequent photoreceptor precursors by adding small molecule inhibitors. Unlike most other groups, they did not choose DKK1 as a common factor to direct ESCs to retinal progenitors and found a high rate of neural tumor formation following ocular injection as a result (5). Thus, a more in-depth comparison between P-RPCs and ESC-RPCs, such as assessment of epigenetic signatures using genome-wide tools (11), will be required to optimize differentiation conditions and to make progress toward translational applications.

As an alternative to ESC-RPCs or P-RPCs for cell therapy, pluripotent stem cells could be differentiated into more mature, lineage-restricted stem cells, such as neural stem cells, to reduce tumorigenicity (12). However, as Cui et al. discuss, donor cells might lose desired functions and the ability to integrate to host retina by prolonged differentiation. Thus, it will be critical to identify the appropriate stage of ESC-derived cells and ensure the balance between safety and efficiency (13). It remains to be 
seen how broadly the approach to retinal progenitor cell differentiation by the WNT pathway modulation can be applied to differentiation of other tissue or cell types. Nevertheless, predifferentiation of ESCs in vitro to a desired cell population before transplantation both to ensure efficiency of transplantation as well as to minimize the risk of tumor formation may be a good general strategy in stem cell therapy.

\section{Acknowledgments}

This work was supported by 973 Program grants (2013CB967504); NSFC grants (no. 81270992 and no. 81130017); NEI/NIH grants EY014428, EY018660, EY019270, EY021374; VA Merit Award; and the Burroughs Wellcome Fund Clinical Scientist Award in Translational Research.

Address correspondence to: Yehong Zhuo, State Key Laboratory of Ophthalmology, Zhongshan Ophthalmic Center,
Sun Yat-sen University, 54 South Xianlie Road, Guangzhou, 510060, China. Phone: 86.20.87330371; Fax: 86.20.87333271; E-mail: zhuoyh@mail.sysu.edu.cn. Or to: Kang Zhang, Institute for Genomic Medicine, University of California, San Diego, Department of Ophthalmology and Shiley Eye Center, 9415 Campus Point Drive, La Jolla, California 92093-0946, USA. Phone: 858.246.0814; Fax: 858.246.0873; E-mail: kang.zhang@gmail.com.

1. Evans MJ, Kaufman MH. Establishment in culture of pluripotential cells from mouse embryos. Nature. 1981;292(5819):154-156.

2. Doi $\mathrm{D}$, et al. Prolonged maturation culture favors a reduction in the tumorigenicity and the dopaminergic function of human ESC-derived neural cells in a primate model of Parkinson's disease. Stem Cells. 2012;30(5):935-945.

3. Lamba DA, Gust J, Reh TA. Transplantation of human embryonic stem cell-derived photoreceptors restores some visual function in Crx-deficient mice. Cell Stem Cell. 2009;4(1):73-79.

4. Schwartz SD, et al. Embryonic stem cell trials for macular degeneration: a preliminary report. Lancet. 2012;379(9817):713-720.
5. Cui L, et al. WNT signaling determines tumorigenicity and function of ESC-derived retinal progenitors. J Clin Invest. 2013;123(4):1647-1661.

6. Przyborski SA. Differentiation of human embryonic stem cells after transplantation in immune-deficient mice. Stem Cells. 2005;23(9):1242-1250.

7. Kielman MF, et al. Apc modulates embryonic stem-cell differentiation by controlling the dosage of beta-catenin signaling. Nat Genet. 2002; 32(4):594-605

8. Meyer JS, et al. Modeling early retinal development with human embryonic and induced pluripotent stem cells. Proc Natl Acad Sci U S A. 2009; 106(39):16698-16703

9. Osakada F, Ooto S, Akagi T, Mandai M, Akaike A, Takahashi $\mathrm{M}$. Wnt signaling promotes regeneration in the retina of adult mammals. J Neurosci. 2007; 27(15):4210-4219.

10. Zhang K, Ding S. Stem cells and eye development. NEng J Med. 2011;365(4):370-372.

11. Hannum G, et al. Genome-wide methylation profiles reveal quantitative views of human aging rates. Mol Cell. 2013;49(2):359-367.

12. Li W, et al. Rapid induction and long-term self-renewal of primitive neural precursors from human embryonic stem cells by small molecule inhibitors. Proc Natl Acad Sci U S A. 2011;108(20):8299-8304.

13. West EL, et al. Defining the integration capacity of embryonic stem cell-derived photoreceptor precursors. Stem Cells. 2012;30(7):1424-1435.

\title{
Striking the target in iron overload disorders
}

\author{
Karin E. Finberg
}

Department of Pathology, Duke University Medical Center, Durham, North Carolina, USA.

\begin{abstract}
The liver, a major site of body iron stores, mediates key responses that preserve systemic iron homeostasis. In this issue of the JCI, Guo et al. demonstrate that administration of antisense oligonucleotides that reduce expression of Tmprss6, a hepatic protein that plays an essential role in maintaining iron balance, can attenuate disease severity in mouse models of human iron overload disorders. These data reveal the potential of novel TMPRSS6-targeted therapies for the treatment of clinical conditions such as hereditary hemochromatosis and $\beta$-thalassemia.
\end{abstract}

\section{Hepcidin and the regulation of systemic iron balance}

The majority of iron required daily by the adult human body is used to meet the demands of hemoglobin synthesis. Most of this iron is obtained through the recycling of senescent erythrocytes by macrophages in the spleen, liver, and bone marrow, while a small amount is absorbed from the diet in the duodenum. Hepcidin, a small circulating peptide released by the liver, regulates iron balance by limiting both the absorption of iron from the diet and the release of iron from macrophage stores (1).

Conflict of interest: The author has declared that no conflict of interest exists.

Citation for this article: J Clin Invest. 2013; 123(4):1424-1427. doi:10.1172/JCI68889.
Hepcidin mediates these effects by triggerferroportin, a cellular iron exporter that is highly expressed at the basolateral membrane of enterocytes and the cell membrane of macrophages (Figure 1). In hepatocytes, hepcidin transcription is modulated by an intracellular signaling cascade that is activated by binding of BMP ligands to a cellsurface receptor complex (Figure 2). The liver, a major site of iron storage, increases production of the BMP family member BMP6 in response to rising local iron stores; this leads to increased signaling for hepcidin production, which in turn limits further dietary iron absorption. Appropriate regulation of intestinal iron absorption is critical, as there is no regulated mechanism for eliminating surplus iron from the body. ing the internalization and degradation of

\section{Hepcidin insufficiency in iron overload disorders}

Inherited forms of iron overload (hemochromatosis) result from mutations in gene products that are required locally in the liver for hepcidin production. In these disorders, the resulting hepcidin insufficiency leads to gastrointestinal iron absorption that exceeds the body's needs. The accumulation of excess iron promotes oxidative damage to tissues, which can ultimately lead to failure of organs such as the heart, liver, and endocrine glands. Hepcidin levels are inappropriately low relative to body iron stores in another class of clinical disorders associated with systemic iron loading: congenital anemias that are characterized by ineffective erythropoiesis (IE) (2). IE describes a defective form of erythroid maturation characterized by an increased proportion of erythroid precursors, which, due to excessive apoptosis, fail to produce the normal complement of mature erythrocytes. In $\beta$-thalassemia, the most common inherited form of IE, the primary genetic defect leads to reduced synthesis of the $\beta$-globin component of adult hemoglobin. The result is an excess 